\title{
How to Capture the Bleeding Phenotype in FXI- Deficient Patients
}

\author{
Debora Bertaggia Calderara ${ }^{1}$ Maxime G. Zermatten ${ }^{1} \quad$ Alessandro Aliotta $^{1} \quad$ Lorenzo Alberio $^{1}$ \\ ${ }^{1}$ Division of Hematology and Central Hematology Laboratory, \\ Lausanne University Hospital (CHUV) and University of Lausanne \\ (UNIL), Lausanne, Switzerland \\ Address for correspondence Debora Bertaggia Calderara, PhD, \\ Hemostasis and Platelet Research Laboratory, Rue du Bugnon 27 SUD, \\ $\mathrm{CH}-1011$ Lausanne, Switzerland \\ (e-mail: debora.bertaggia-calderara@chuv.ch).
}

Hämostaseologie 2020;40:491-499.

Abstract
Keywords
- coagulation factor XI
- FXI deficiency
- global assays
- thrombin generation
- hemostatic potential
Factor $\mathrm{XI}(\mathrm{FXI})$ is a serine protease involved in the propagation phase of coagulation and in providing clot stability. Several mutations in the F11 gene lead to FXI deficiency, a rare mild bleeding disorder. Current laboratory methods are unable to assess bleeding risk in FXI-deficient patients, because the degree of bleeding tendency does not correlate with plasma FXI activity as measured by routine coagulometric aPTT-based assays. Bleeding manifestations are highly variable among FXI-deficient patients and FXI replacement therapy can be associated with an increased thrombotic risk. A correct evaluation of the patient hemostatic potential is crucial to prevent under- or overtreatment. In recent years, different research groups have investigated the use of global coagulation assays as alternative for studying the role of FXI in hemostasis and identifying the clinical phenotype of FXI deficiency. This brief review article summarizes the main features of coagulation factor XI and its deficiency and resumes the principle axes of research and methods used to investigate FXI functions.

\section{Introduction}

Plasma protein coagulation factor XI (FXI) is the zymogen of the coagulation protease FXIa, which contributes to physiological hemostasis and is involved in pathological thrombosis. ${ }^{1,2}$ Originally, FXI was considered part of the contact activation pathway. In presence of negatively charged surfaces, prekallikrein, and high-molecular-weight kininogen, FXII becomes activated (FXIIa) and activates FXI. ${ }^{3,4}$ Activated FXI (FXIa) promotes thrombin generation (TG) and subsequent fibrin formation through FIX activation. Contact activation is the basis for the activated partial thromboplastin time (aPTT) assay. However, FXIa sustains in vivo TG independently from FXIIa, ${ }^{5}$ participating in the tissue factor (TF)-independent propagation phase of coagulation. ${ }^{4}$ Here, FXI is activated in a positive feedback loop by small amounts of thrombin generated in the initial phase of coagulation via TF/FVIIa complex. FXIa increases TG through the activation of FIX (-Fig. 1). ${ }^{1,4,6}$ Of note, when FXII is activated in vivo by negative surfaces, such as polyphosphates, collagen or nucleic acids, subsequent FXI activation promotes downstream coagulation. ${ }^{2,3}$ FXI is primarily produced by hepatocytes ${ }^{7,8}$ and circulates in human plasma as a complex with high-molecular-weight kininogen, ${ }^{9}$ which helps the interaction of FXI with activated platelets ${ }^{10}$ via glycoprotein (GP) Ib receptor. ${ }^{11}$ FXI has a unique structure among the coagulation factors, since it is a disulfide-linked homodimer consisting of two identical subunits of $80 \mathrm{kD}$. Each subunit is composed of four apple domains (A1-A4) and one catalytic domain. ${ }^{1}$ In addition to thrombin, FXI can be activated by FXIla or by FXIa (autoactivation). ${ }^{4,6}$ FXI is converted to the active form by cleavage after residue 369 (Arg369-Ile370), which induces a conformational change in the catalytic domain, leading to the active form FXIa. ${ }^{12}$ The activation of FXI by thrombin or FXIla goes through the formation of an intermediate form, named 1/2-FXIa, in which only one of the two subunits is activated. ${ }^{13}$ It has been proposed that the dimeric structure is necessary to localize FXI to the platelet surface: the intermediate form 1/2-FXIa binds to GPIb receptor through the A3 domain of the non-activated subunit, while the activated subunit would be free to bind its substrate FIX. ${ }^{13}$ received

April 30, 2020

accepted after revision

August 25, 2020 (c) 2020 Georg Thieme Verlag KG Stuttgart · New York
DOI https://doi.org/

10.1055/a-1227-8122. ISSN 0720-9355. 


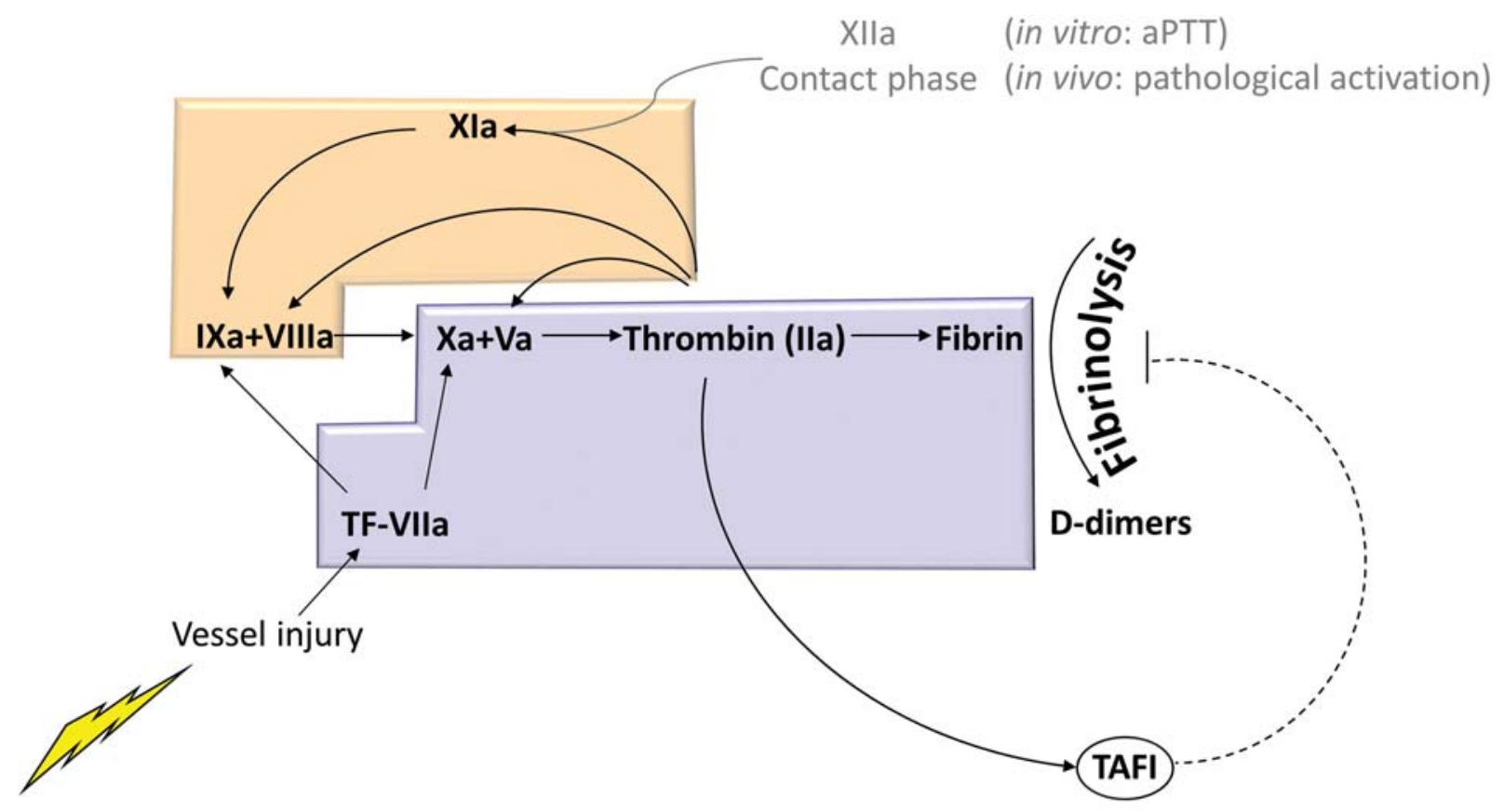

\section{TF dependent : $1^{\text {st }}$ wave of TG and fibrin deposition}

\section{TF independent $: 2^{\text {nd }}$ wave of TG and fibrin deposition}

Fig. 1 Simplified scheme of coagulation cascade and fibrinolysis (adapted from Bouma et al ${ }^{59}$ and Vandenbroucke et al ${ }^{60}$ ): Exposure of tissue factor (TF) to blood after vascular injury leads to TF-VIla complex formation. TF-VIla complex activates factors X and IX to factors Xa and IXa at the site of the injury, triggering the initiation stage of blood coagulation. Factor Xa generates a small amount of thrombin and the first deposition of fibrin fibers occurs (TF-dependent coagulation). Thrombin produced in this initial phase of coagulation activates platelets and factors V, VIII, and XI with positive feedback loops, which substantially increase thrombin production, leading to the second wave of thrombin generation and fibrin deposition (amplification/propagation). This secondary burst of thrombin is required for a normal hemostatic response and for regulation of the fibrinolytic system. Thrombin stabilizes clots activating TAFI, which protects the clot from fibrinolysis. Note that FXI can be activated either by FXIla as part of contact activation pathway (i.e., in vitro in the aPTT assay or in vivo in pathological conditions ${ }^{61}$ ) or directly by thrombin in the TF-independent amplification/propagation phase of coagulation in vivo. aPTT, activated partial thromboplastin time; TAFI, thrombin-activatable fibrinolysis inhibitor; TF, tissue factor; TG, thrombin generation.

FXI has multiple in vivo roles in hemostasis, some of which are not fully elucidated. These roles can be summarized in three different actions. (1) To sustain TG by enhancing TF-independent propagation of the clotting cascade ( - Fig. 1). ${ }^{4,6,14,15}$ (2) To downregulate fibrinolysis: the additional thrombin produced through the FXI feedback-loop after clot formation activates thrombin-activatable fibrinolysis inhibitor (TAFI), protecting clots from fibrinolysis. ${ }^{16}(3)$ To increase procoagulant activity through inactivation of tissue factor pathway inhibitor. ${ }^{17}$

\section{FXI Deficiency and Bleeding}

Reduced activity of FXI characterizes FXI deficiency, a mild to moderate bleeding diathesis, previously known as hemophilia C. This rare autosomal bleeding disorder affects both genders equally and has a frequency of 1:1,000,000 worldwide, with a higher rate in people of Ashkenazi Jewish heritage (1:450). ${ }^{18,19}$ In Europe, a recent large genetic study has reported a frequency of 12.9 affected people in a million. ${ }^{20} \mathrm{~A}$ large variety of mutations ${ }^{21}$ has been reported within the $F 11$ gene, which is localized on chromosome 4. The large majority of these mutations lead to FXI deficiency type I, affecting both FXI activity and antigen, while few others lead to FXI deficiency type II, affecting only FXI activity (FXI database: http://www.factorxi.org ${ }^{22}$ ). FXI deficiency has a peculiar clinical interest: bleeding symptoms are very heterogeneous among patients ${ }^{23}$ and do not correlate with FXI residual plasma level or with genetic phenotype. ${ }^{24}$ Differently from hemophilia A and B, in FXI deficiency spontaneous bleeding is uncommon and mostly occurs after trauma or surgery, especially in tissues with high fibrinolytic activity (oral, nasal cavity, and urinary tract). ${ }^{24,25}$ Although bleeding tendency does not correlate with FXI coagulant activity (FXI:C, normal range: 70-150 U/dL), FXI deficiency can be classified based on these values as partial (FXI:C between 20 and $70 \mathrm{U} / \mathrm{dL}$ ) or severe (FXI:C $<20 \mathrm{U} / \mathrm{dL}$ ). ${ }^{26,27}$ Diagnosis of FXI is based on family history or presurgery laboratory workup and completed by routine laboratory methods (prothrombin time [PT], aPTT, FXI:C) and in addition by genetic analysis. Patients with FXI deficiency will usually have a prolonged aPTT and normal PT. ${ }^{28,29}$ However, 
patients with mild deficiency might have normal aPTT. ${ }^{28}$ FXI-deficient patients are treated on demand or prior planned interventions and current treatments consist of antifibrinolytics, desmopressin, ${ }^{30}$ virus-inactivated plasma, and plasma-derived FXI concentrates. ${ }^{31}$ The last one carries an increased risk of thrombosis and should be administered with precaution. ${ }^{29}$

\section{Laboratory Methods to Assess FXI Function}

aPTT-based assays are not reliable for predicting bleeding tendency in FXI-deficient patients ${ }^{32}$ or thrombotic risk after FXI replacement therapy, because they provide a limited view of the hemostatic system. ${ }^{32}$ In fact, routine coagulation assays reflect only the amount of thrombin required to signal the onset of clotting. The amount of thrombin needed to produce the initial fibrin formation is very small $(<5 \%$ of the total thrombin potential) and it is generated during the initial phase of coagulation. The major burst of thrombin occurs in vivo after the initial fibrin formation and it is used to increase and consolidate the clot. ${ }^{33}$ FXI coagulant activity (FXI:C) depends on the amount of FXI and-in case of preanalytical coagulation activation-of FXIa present in plasma. ${ }^{34,35}$ This amount is estimated by the ability of an unknown plasma to correct the aPTT of a FXI-depleted plasma. The test is triggered by the contact activation pathway, which generates FXIla, leading to the activation of FXI to FXIa and TG. The endpoint of the test is the formation of fibrin, which occurs rapidly, before achieving the full TG potential. FXI plays a role in sustaining TG after clot formation as well, increasing clot stability and resistance to fibrinolysis. ${ }^{36}$ FXI activity as measured by aPTT-based assay does not reflect the physiological activity of FXI and does not predict bleeding in FXI deficiency. This is explained by the fact that in the aPTT assay FXI is exclusively activated by FXIIa as a part of the contact activation pathway (a process which is not required in vivo for normal hemostasis as demonstrated by the absence of bleeding diathesis in FXII deficiency ${ }^{37}$ ). In addition, the activation of FXI by thrombin, which occurs in vivo on platelet surface, is not covered by this clotting test. Thus, it is unlikely that aPTT-based FXI activity might reflect FXI's physiological role and correlate with bleeding phenotype. ${ }^{32}$

The lack of conventional assays able to discriminate FXI clinical phenotype has prompted researchers to investigate whether global coagulation and/or fibrin clot formation assays could be better tools for predicting bleeding risk ( - Table 1). Global coagulation assays measure physiological aspects of the coagulation process (e.g., the amount of thrombin produced, the firmness/structure of fibrin clot or its resistance to fibrinolysis) and consider several components of the coagulation cascade rather than the plasma level of a single coagulation factor. Thus, global assays reflect better organization of the hemostatic system in vivo, which reminds more of a network than of separate pathways of activation.

Till this date different studies have used global coagulation assays to investigate FXI clinical phenotype and/or FXI replacement therapy (summarized in -Table 1 ). Different axes of research/methods investigating FXI functions using global assays can be outlined.

\section{Thrombin Generation Assay-Calibrated Automated Thrombogram}

Thrombin is the key enzyme of the coagulation cascade, since it cleaves fibrinogen into fibrin, which is needed for platelet clot stabilization and to prevent bleeding. Thrombin also regulates the coagulation cascade through a set of positive and negative feedback mechanisms (- Fig. 1). TG assays allow to continuously monitoring thrombin production in clotting plasma. The reference method is the calibrated automated thrombogram (CAT), a semiautomated method developed by Hemker et al. ${ }^{38}$ The test is performed in either platelet-poor (PPP) or plateletrich plasma (PRP). The coagulation reaction is triggered by TF, requires calcium and-when testing $\mathrm{PPP}-$ phospholipids. TF concentration is variable depending on the condition/disease analyzed. TF is constantly present and uniformly distributed in the sample mixture; thus, CAT is considered a homogeneous model of coagulation. The assay uses a modified fluorogenic substrate (Z-Gly-Gly-Arg-7-amino-4-methylcoumarin) which is cleaved by thrombin and produces fluorescence proportionally to the amount of thrombin generated. By means of a thrombin calibrator, the fluorescence of the unknown sample is converted to the concentration of thrombin, thus constructing a TG curve ( - Fig. 2A).

Identification of FXI clinical phenotype by CAT assay has produced conflictual results (summarized in - Table 1). ${ }^{39-45}$ This may be explained by the different criteria used to classify patients in bleeders or non-bleeders and by the various experimental conditions used to measure TG. Standard bleeding scores are inadequate for predicting bleeding risk in FXIdeficient patients. ${ }^{46}$ Thus, it is possible that in the studies that were unable to separate bleeders from non-bleeders, the patients were inappropriately phenotyped, either because the classification was based on a standard bleeding score or because the patients were not exposed to an appropriate hemostatic challenge (e.g., interventions in high fibrinolytic area, such as tonsillectomy or dental extractions). In addition, studies listed in - Table 1 used very different experimental conditions: (1) presence or absence of platelets; (2) presence or absence of corn trypsin inhibitor (CTI); and presence of different concentrations of TF. This variety makes data comparison among studies quite difficult. Here, we summarize the main study characteristics and results (see also - Table 1 ).

The studies from Zucker et $\mathrm{al}^{39}{ }^{39}$ Guéguen et $\mathrm{al},{ }^{40}$ and Bertaggia Calderara et $\mathrm{al}^{44}$ found that TG measured by CAT assay in PPP using TF at $1 \mathrm{pM}$ could not discriminate between FXI-deficient bleeder and non-bleeder individuals. Of note, Zucker et $\mathrm{al}^{39}$ used CTI in collecting tubes to prevent contact activation, while Guéguen et $\mathrm{al}^{40}$ and Bertaggia Calderara et $\mathrm{al}^{44}$ did not.

Livnat et $\mathrm{al}^{43}$ performed TG assay in PPP with $1 \mathrm{pM}$ TF as a trigger or without TF, in the presence or absence of CTI. They found that TG parameters (endogenous thrombin potential [ETP] and peak height) were both significantly decreased in citrated plasma, only when coagulation was induced by recalcification (without addition of TF). The absence of significant difference observed between FXI bleeders and non-bleeders in the presence of CTI might be due to a concentration of CTI too high in the collection tubes, which could have inhibited FXIa. ${ }^{47}$ 


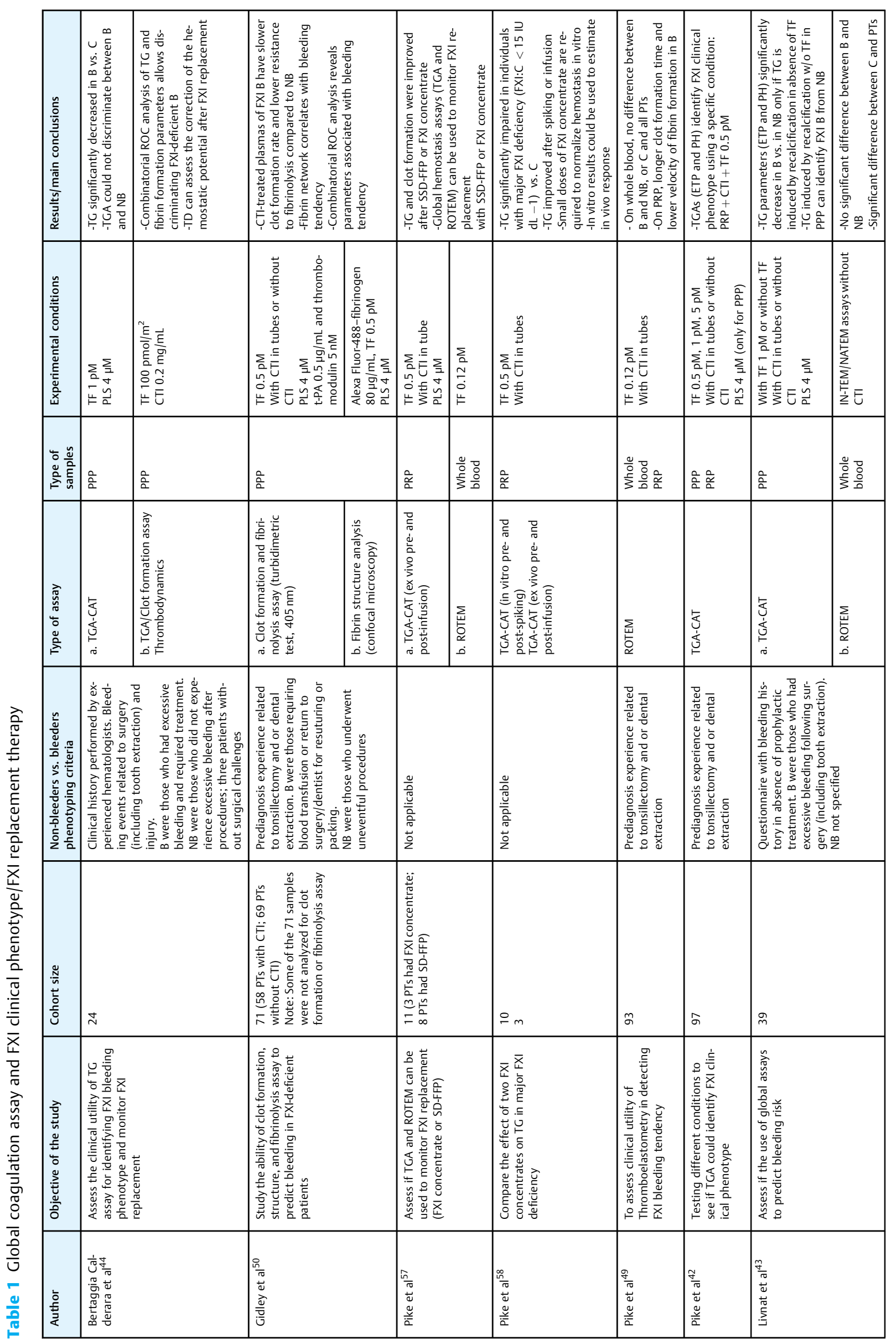




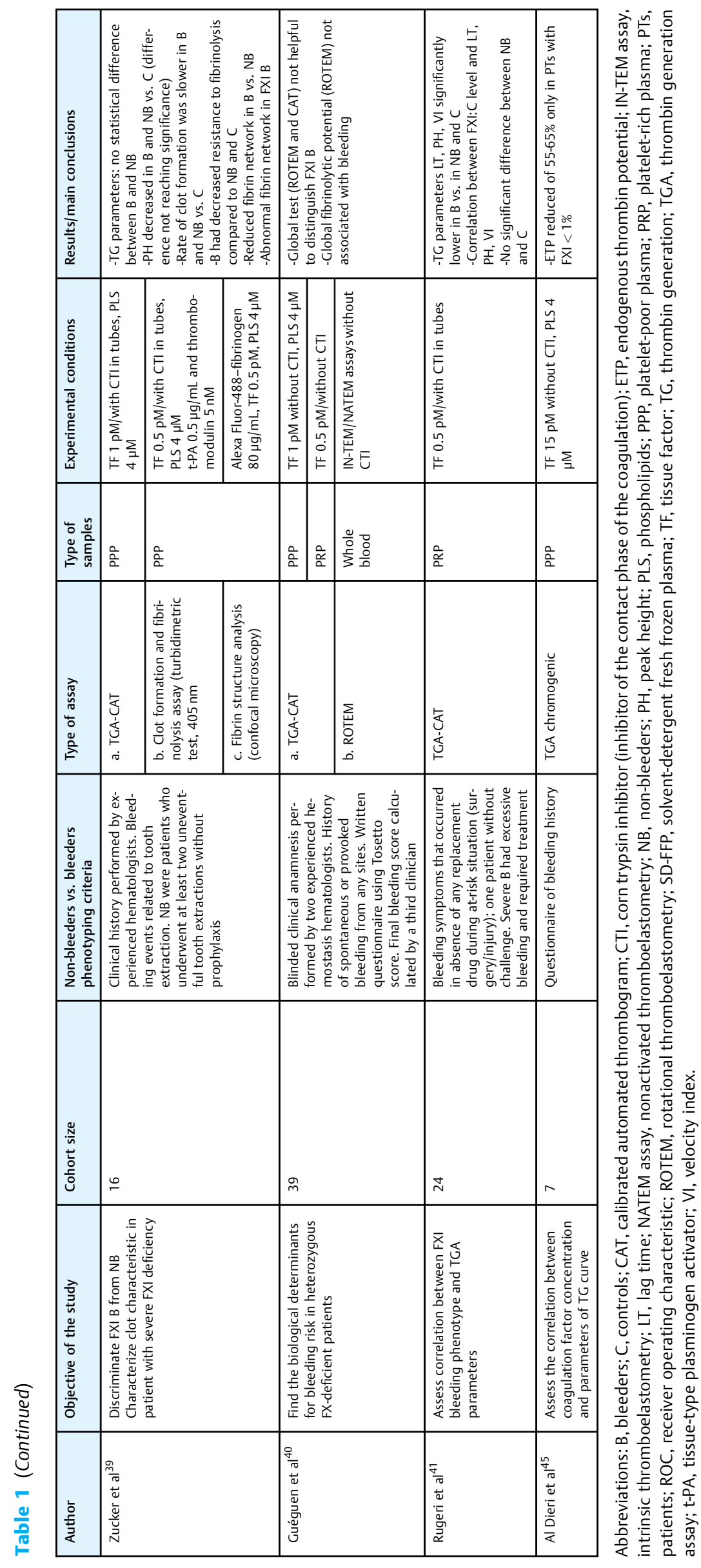


Al Dieri et $\mathrm{al}^{45}$ investigated TG in FXI deficiency and others rare bleeding disorders in PPP. In a small cohort of seven patients, they found a 55 to $65 \%$ reduction of the ETP only in patients with FXI $<1 \%$, while the others (FXI between 1 and 5\%) had normal TG. However, the very high TF concentration used (15 pM) probably limited the sensitivity of the TG assay. ${ }^{47,48}$

Two studies ${ }^{41,42}$ performed TG measurements in PRP. Blood was collected in tubes containing CTI and TG assay was done using lower concentration of TF $(0.5 \mathrm{pM})$. Rugeri et $\mathrm{al}^{41}$ studied a cohort of 24 FXI-deficient patients and found that TG parameters (lag time/peak height/velocity index) were significantly different in bleeders versus non-bleeders, while there was no difference in non-bleeders versus controls. Pike et $\mathrm{al}^{42}$ tested several sample conditions (i.e., PPP or PRP, with or without CTI, different TF concentrations) in a larger cohort of patients $(n=97)$. Among the different conditions tested, TG measured in PRP in the presence of CTI best differentiated between bleeders and non-bleeders, as it was confirmed by receiver operating characteristic (ROC) curve analysis (specificity of $80 \%$ for ETP and of $67 \%$ for peak height ${ }^{42}$ ).

While the use of PPP is very convenient because it is easy to standardize and allows working with frozen samples from different laboratories, the use of PRP covers the role of GPIb and the contribution of activated platelets in FXI activation. ${ }^{6}$ Nevertheless, PRP samples cannot be stored and are more difficult to handle, since platelets can be unintentionally activated. In sum, these studies have shown that the use of a low TF concentration $(<1 \mathrm{pM})^{48}$ in the presence of CTI to inhibit the contact activation pathway (which enhances TG via propagation loop coagulation of factors XI and IX/VIII) is important for increasing the sensitivity of TG assay to FXI clinical phenotype.

\section{Clot Formation and Fibrinolysis Assay}

Further studies have used rotational thromboelastometry $(\text { ROTEM })^{40,43,49}$ or turbidimetric assay ${ }^{39,50}$ ( - Fig. 2B, C). These studies focused on clot formation and fibrinolysis to investigate the hemostatic role of FXI. Thromboelastometry is generally performed in citrated whole blood and the assay provides a graphic representation of clot formation, stabilization, and fibrinolysis. The few studies performed $40,43,49$ concluded that ROTEM performed in whole blood was not able to discriminate FXI-deficient bleeders from FXI nonbleeders, independently from the presence of CTI. Of note, Pike et $\mathrm{al}^{49}$ found that if the assay was performed in PRP + CTI, FXI-deficient bleeders had a longer clot formation time with decreased velocity of fibrin generation, which is in agreement with studies using other assays. ${ }^{39,41,44}$

Turbidimetric assay is a quite accessible technique and is performed in citrated CTI-treated PPP, in the presence of low TF concentration and recalcification. The assay can be done in the presence of tissue plasminogen activator (tPA) to monitor fibrinolysis; readings are done in a plate reader with absorbance at $405 \mathrm{~nm}$ and monitoring of turbidity allows building a clot formation/fibrinolysis curve, characterized by several parameters (onset of clot formation, clot formation rate, time to peak, peak turbidity change, and area under the curve). Zucker et $\mathrm{al}^{39}$ in a small cohort of 16 patients found that FXI- deficient patients had a slower rate of clot formation compared to controls and that clot from FXI bleeders was significantly less resistant to fibrinolysis compared to that from nonbleeders and controls. Gidley et $\mathrm{al}^{50}$ in a larger cohort of patients $(n=71)$ confirmed these observations. The authors found that in FXI-deficient bleeder plasma treated with CTI, the clot had a significantly slower formation rate and that it was less resistant to fibrinolysis compared to non-bleeders and controls. They proposed that using CTI-treated plasma, the association of aPTT with parameters of fibrinolysis assays (clot formation rate and area under the curve) in a combined model could help detect FXI-deficient bleeders. Interestingly, both studies $^{39,50}$ were completed by confocal images of fibrin network structure observed in plasma clots. Zucker et $\mathrm{al}^{39}$ observed that fibrin network in clots from bleeders was reduced by about 20 to $25 \%$ compared with both controls $(p<0.05)$ and non-bleeders $(p<0.02)$. Gidley et $\mathrm{al}^{50}$ found that fibrin network density in clots from FXI-deficient bleeders trended toward a reduced density compared to controls and non-bleeders. These studies ${ }^{39,50}$ highlight the role of clot density and its stability toward fibrinolysis in FXI-deficient bleeders. ${ }^{32}$ Of note, Colucci et al $^{51}$ studied TG and fibrinolytic resistance in a cohort of 18 patients exhibiting various degree of FXI deficiency. Their work suggested that the reduced fibrinolytic resistance observed in clots from FXI-deficient patients might be due to a defective TAFI-dependent inhibition of fibrinolysis, described as "TAFla resistance." ${ }^{51}$ While the underlying mechanism of TAFIa resistance needs to be further elucidated, this activity may be important to understand the variety of the bleeding tendency in FXI deficiency. ${ }^{32,52}$

\section{Thrombin Generation and Clot Formation in a Spatial Heterogeneous Model of Coagulation- Thrombodynamics Assay}

Thrombodynamics (TD) assay ( - Fig. 2D), a video microscopy system recently marketed, is an alternative experimental model of coagulation, which considers the spatial and temporal dynamic of coagulation and the biochemical reaction of the coagulation cascade. The test is performed on recalcified PPP and the coagulation is triggered by a thin layer of TF immobilized on a coated surface at low concentration (density $=100$ $\mathrm{pmol} / \mathrm{m}^{2}$ ) in the presence of CTI, phospholipids, and a modified fluorescent substrate cleavable by thrombin. The test allows monitoring simultaneously TG and fibrin clot formation. ${ }^{53-56}$ In this experimental model, coagulation is triggered by immobilized TF and propagates into the bulk of plasma, where TF is absent, mimicking a blood vessel damage. Images of TG and clot formation are recorded by a dedicated software, which calculates several parameters of the TF-dependent and -independent phases of coagulation. Thus, this system is suitable to analyze FXI's role in the coagulation. In fact, it is performed in the presence of CTI, which increases the sensitivity of the test to the amount of thrombin generated via the loop FXI and VIII/ IX. Furthermore, with increasing distance from the TF-coated surface, the influence of TF on the coagulation cascade becomes null and the assay can measure parameters of TG and clotting formation of the TF-independent phase of coagulation in which FXI plays a pivotal role. Using this assay in a 


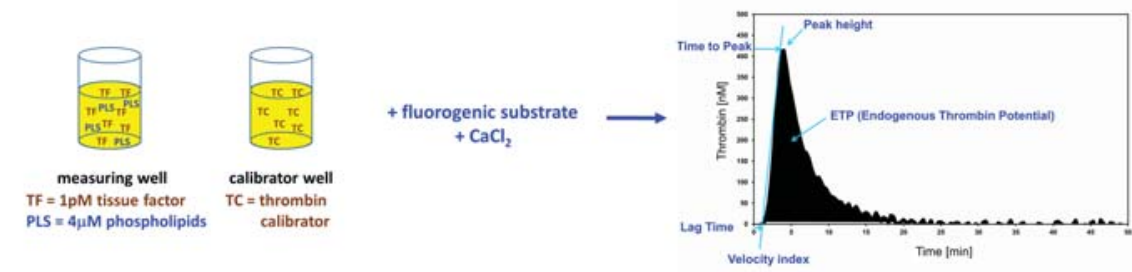

B ROTEM`ASSAY
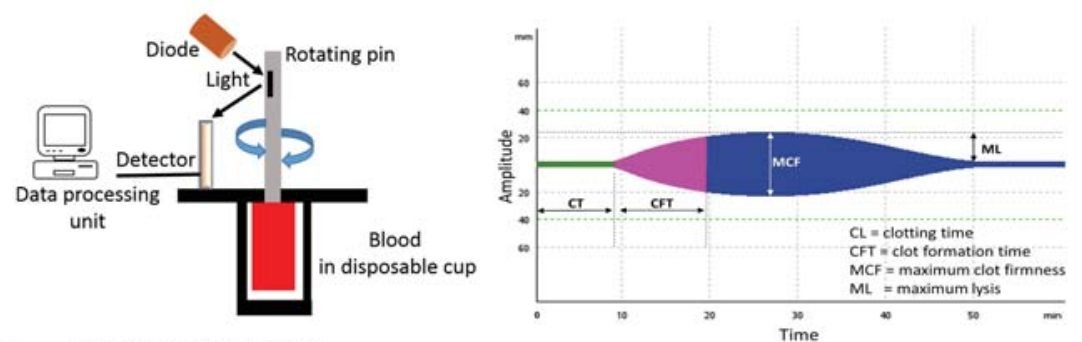

C TURBIDIMETRIC ASSAY

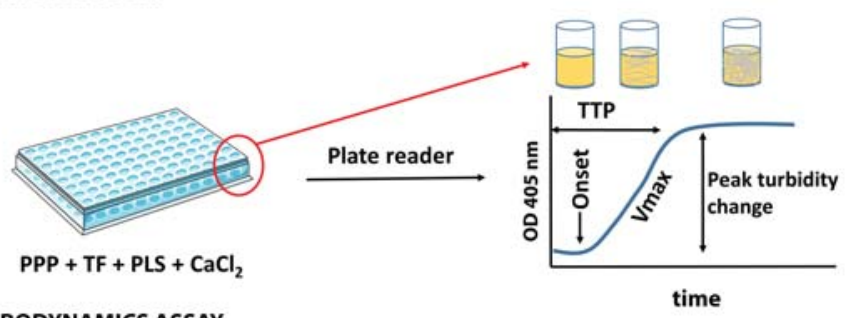

D THROMBODYNAMICS ASSAY
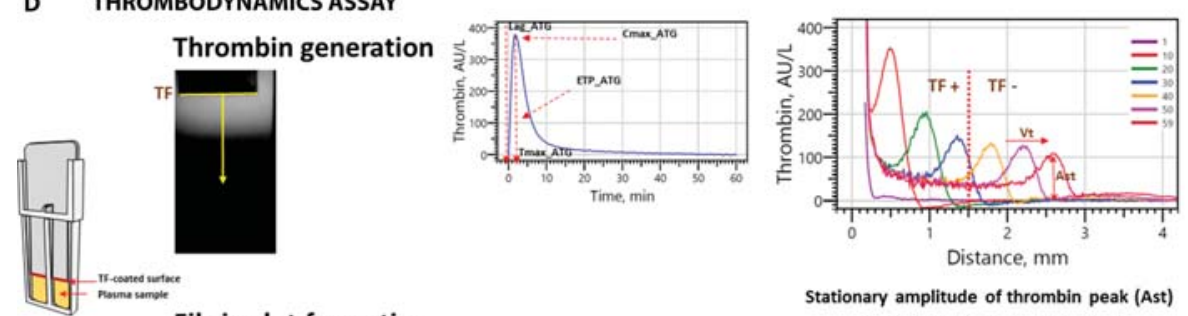

Fibrin clot formation

Stationary amplitude of thrombin peak (Ast)

Rate of thrombin propagation (Vt)
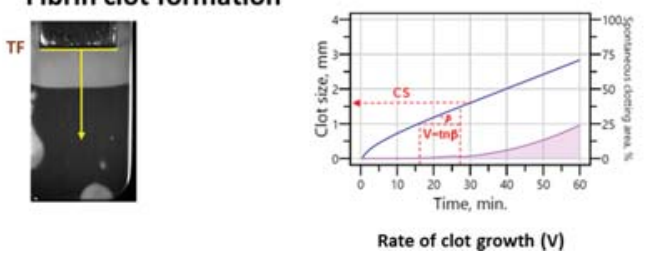

Fig. 2 Basic principles of different global coagulation assays. (A) In calibrated automated thrombogram (CAT) assay, tissue factor (TF) is constantly present and equally distributed in the sample mixture (measuring well). Thrombin generation curve is described by (1) the lag time, which is the time between the addition of the trigger and generation of thrombin; (2) the time to peak, which is the time needed to reach the maximal amount of thrombin; (3) the peak height which is the maximal amount of thrombin generated; (4) the velocity index which is the slope of the thrombin generation curve, reflecting the rate at which thrombin is generated; and (5) the endogenous thrombin potential, which is the area under the curve representing the overall amount of thrombin generated. (B) In rotational thromboelastometry (ROTEM), citrated whole blood is added in a disposable cartridge together with calcium and an activator. A pin, suspended in the sample, oscillates over a small arc. As the blood starts clotting, the raising clot firmness increasingly restricts the rotation of the pin. This movement is detected and charted. A depiction of highly pathological ROTEM output illustrates the parameters of the measure: clotting time (CT), clot formation time (CFT), maximum clot firmness (MCF), and maximum lysis (ML). (C) A turbidimetric assay measures clot properties by reading the optical density of the forming clot over time. An activation mix containing TF, calcium, and phospholipids is added to a 96-wells plate containing plasma samples and coagulation is triggered. The samples are measured in a plate reader at absorbance of $405 \mathrm{~nm}$ over time. The optical density measures are plotted versus time. Onset: time to the inflection point before turbidity increases; time to peak (TTP), is the time needed to reach the plateau; peak turbidity change is the maximum clot turbidity less the initial turbidity; Vmax is the rate of clot formation. The same principle can be used to monitor clot lysis, after addition of t-PA to the plasma (not shown in the figure; - Fig. $2 \mathrm{C}$ was created using images from the Servier Medical Art, which are licensed under a Creative Commons Attribution 3.0 Unported License; https://smart.servier.com). (D) Thrombodynamics assay (TD) is a spatial heterogeneous system. TF is immobilized to a surface and coagulation spreads from the TF-coated surface to the bulk of plasma where the TF is absent. Differently from the CAT assay, the TD assay will measure parameters informative of the thrombin generated during the TF-independent phase of coagulation (Ast = amplitude of the thrombin moving peak; $\mathrm{Vt}$ = rate of thrombin propagation) and parameters describing clot formation (clot growth and clot size). TF +, presence of tissue factor; TF - , absence of tissue factor. 
cohort of 24 FXI-deficient individuals, Bertaggia Calderara et $\mathrm{al}^{44}$ could observed that FXI-deficient bleeders were characterized by a significant lower TG and clot formation rate in the TF-independent phase of coagulation. An algorithm based on combinatorial ROC analysis of TG and fibrin clot formation parameters could identify all of the FXI-deficient patients with bleeding phenotype and $82 \%$ of FXI patients without bleeding symptoms. In sum, the spatial heterogeneous model of coagulation appears to improve the ability of traditional TG assays to recognize the FXI bleeding phenotype, offering a promising tool for a tailored treatment of the FXI-deficient patient.

\section{Global Assay for Monitoring FXI Replacement}

Global TG assays have been used to assess hemostatic potential after FXI replacement with FXI concentrates. ${ }^{44,57,58}$ In general, these studies showed that after in vitro spiking of FXI-deficient plasma or after infusion of FXI concentrate in patients, TG was improved. Of note, after the infusion of FXI concentrate, TF-independent parameters measured ex vivo by TD assay shifted toward hypercoagulation at levels of FXI of about $30 \% .{ }^{44}$ These studies ${ }^{44,57,58}$ agreed on the fact that low doses of FXI concentrate were sufficient to normalize hemostasis in vitro and ex vivo ${ }^{44,58}$ concluding that global TG assays are a promising tool for monitoring FXI replacement.

\section{Conclusions}

The diagnosis and management of FXI-deficient patients remain challenging. Development of global coagulation assays as alternative methods of testing has increased the sensitivity and ability to correlate to some extent TG or clot formation parameters to FXI bleeding phenotype. However, further prospective studies in large cohorts of patients are needed to use these methods clinically. Further investigation is needed to fully understand the complex mechanism underlying FXI deficiency bleeding phenotype and FXI's role in hemostasis.

\section{Conflict of Interest}

The authors declare that they have no conflict of interest.

\section{Acknowledgements}

The authors would like to thank Ms. Christine Coutaz, deputy head technician, Central Hematology Laboratory, CHUV, Lausanne, for providing the pathological ROTEM output illustrated in - Fig. 2B and Prof. Michel Duchosal for support.

\section{References}

1 Emsley J, McEwan PA, Gailani D. Structure and function of factor XI. Blood 2010;115(13):2569-2577

2 Visser M, Heitmeier S, Ten Cate H, Spronk HMH. Role of factor XIa and plasma kallikrein in arterial and venous thrombosis. Thromb Haemost 2020;120(06):883-993

3 Renné T, Schmaier AH, Nickel KF, Blombäck M, Maas C. In vivo roles of factor XII. Blood 2012;120(22):4296-4303

4 Gailani D, Broze GJ Jr. Factor XI activation in a revised model of blood coagulation. Science 1991;253(5022):909-912
5 Kravtsov DV, Matafonov A, Tucker EI, et al. Factor XI contributes to thrombin generation in the absence of factor XII. Blood 2009;114 (02):452-458

6 Naito K, Fujikawa K. Activation of human blood coagulation factor $\mathrm{XI}$ independent of factor XII. Factor XI is activated by thrombin and factor XIa in the presence of negatively charged surfaces. J Biol Chem 1991;266(12):7353-7358

7 Walker IR, Milner RA, Johnston MA, Rand CA, Neame PB, Hirsh J. Factors XI and XII are low in subjects with liver disease. Dig Dis Sci 1983;28(11):967-970

8 Dzik WH, Arkin CF, Jenkins RL. Transfer of congenital factor XI deficiency from a donor to a recipient by liver transplantation. $\mathrm{N}$ Engl J Med 1987;316(19):1217-1218

9 Thompson RE, Mandle R Jr, Kaplan AP. Association of factor XI and high molecular weight kininogen in human plasma. J Clin Invest 1977;60(06):1376-1380

10 Ho DH, Badellino K, Baglia FA, et al. The role of high molecular weight kininogen and prothrombin as cofactors in the binding of factor XI A3 domain to the platelet surface. J Biol Chem 2000;275 (33):25139-25145

11 Baglia FA, Gailani D, López JA, Walsh PN. Identification of a binding site for glycoprotein Ibalpha in the Apple 3 domain of factor XI. J Biol Chem 2004;279(44):45470-45476

12 Gailani D, Geng Y, Verhamme I, et al. The mechanism underlying activation of factor IX by factor XIa. Thromb Res 2014;133 (Suppl 1):S48-S51

13 Gailani D, Ho D, Sun MF, Cheng Q Walsh PN. Model for a factor IX activation complex on blood platelets: dimeric conformation of factor XIa is essential. Blood 2001;97(10):3117-3122

14 Whelihan MF, Orfeo T, Gissel MT, Mann KG. Coagulation procofactor activation by factor XIa. J Thromb Haemost 2010;8(07): 1532-1539

15 Matafonov A, Cheng Q Geng Y, et al. Evidence for factor IXindependent roles for factor XIa in blood coagulation. J Thromb Haemost 2013;11(12):2118-2127

16 Von dem Borne PA, Bajzar L, Meijers JC, Nesheim ME, Bouma BN. Thrombin-mediated activation of factor XI results in a thrombinactivatable fibrinolysis inhibitor-dependent inhibition of fibrinolysis. J Clin Invest 1997;99(10):2323-2327

17 Puy C, Tucker EI, Matafonov A, et al. Activated factor XI increases the procoagulant activity of the extrinsic pathway by inactivating tissue factor pathway inhibitor. Blood 2015;125(09):1488-1496

18 Peretz H, Mulai A, Usher S, et al. The two common mutations causing factor XI deficiency in Jews stem from distinct founders: one of ancient Middle Eastern origin and another of more recent European origin. Blood 1997;90(07):2654-2659

19 Shpilberg O, Peretz H, Zivelin A, et al. One of the two common mutations causing factor XI deficiency in Ashkenazi Jews (type II) is also prevalent in Iraqi Jews, who represent the ancient gene pool of Jews. Blood 1995;85(02):429-432

20 Asselta R, Paraboschi EM, Rimoldi V, et al. Exploring the global landscape of genetic variation in coagulation factor XI deficiency. Blood 2017;130(04):e1-e6

21 Rimoldi V, Paraboschi EM, Menegatti M, et al. Molecular investigation of 41 patients affected by coagulation factor XI deficiency. Haemophilia 2018;24(02):e50-e55

22 Saunders RE, O'Connell NM, Lee CA, Perry DJ, Perkins SJ. Factor XI deficiency database: an interactive web database of mutations, phenotypes, and structural analysis tools. Hum Mutat 2005;26 (03):192-198

23 Santoro C, Di Mauro R, Baldacci E, et al. Bleeding phenotype and correlation with factor XI (FXI) activity in congenital FXI deficiency: results of a retrospective study from a single centre. Haemophilia 2015;21(04):496-501

24 Bolton-Maggs PH. Factor XI deficiency-resolving the enigma? Hematology (Am Soc Hematol Educ Program) 2009;2009:97-105

25 Salomon O, Steinberg DM, Seligshon U. Variable bleeding manifestations characterize different types of surgery in patients with 
severe factor XI deficiency enabling parsimonious use of replacement therapy. Haemophilia 2006;12(05):490-493

26 Duga S, Salomon O. Congenital factor XI deficiency: an update. Semin Thromb Hemost 2013;39(06):621-631

27 Bauduer F, de Raucourt E, Boyer-Neumann C, et al; French Postmarketing Study Group. Factor XI replacement for inherited factor XI deficiency in routine clinical practice: results of the HEMOLEVEN prospective 3-year postmarketing study. Haemophilia 2015;21(04):481-489

28 Salloum-Asfar S, de la Morena-Barrio ME, Esteban J, et al. Assessment of two contact activation reagents for the diagnosis of congenital factor XI deficiency. Thromb Res 2018;163:64-70

29 Mumford AD, Ackroyd S, Alikhan R, et al; BCSH Committee. Guideline for the diagnosis and management of the rare coagulation disorders: a United Kingdom Haemophilia Centre Doctors' Organization guideline on behalf of the British Committee for Standards in Haematology. Br J Haematol 2014;167(03):304-326

30 Franchini M, Manzato F, Salvagno GL, Montagnana M, Lippi G. The use of desmopressin in congenital factor XI deficiency: a systematic review. Ann Hematol 2009;88(10):931-935

31 Menegatti M, Peyvandi F. Treatment of rare factor deficiencies other than hemophilia. Blood 2019;133(05):415-424

32 Wheeler AP, Gailani D. Why factor XI deficiency is a clinical concern. Expert Rev Hematol 2016;9(07):629-637

33 Mann KG, Brummel K, Butenas S. What is all that thrombin for? J Thromb Haemost 2003;1(07):1504-1514

34 Morrissey JH, Macik BG, Neuenschwander PF, Comp PC. Quantitation of activated factor VII levels in plasma using a tissue factor mutant selectively deficient in promoting factor VII activation. Blood 1993;81(03):734-744

35 Eschwege V, Trillard M, Robert A. Overestimation of plasma level of factor $\mathrm{V}$ coagulant activity due to unrecognised preanalytical coagulation. Thromb Haemost 2004;91(04):827-828

36 Hoffman M, Monroe DM III. A cell-based model of hemostasis. Thromb Haemost 2001;85(06):958-965

37 Ratnoff OD, Colopy JE. A familial hemorrhagic trait associated with a deficiency of a clot-promoting fraction of plasma. J Clin Invest 1955;34(04):602-613

38 Hemker HC, Wielders S, Kessels H, Béguin S. Continuous registration of thrombin generation in plasma, its use for the determination of the thrombin potential. Thromb Haemost 1993;70(04): 617-624

39 Zucker M, Seligsohn U, Salomon O, Wolberg AS. Abnormal plasma clot structure and stability distinguish bleeding risk in patients with severe factor XI deficiency. J Thromb Haemost 2014;12(07): $1121-1130$

40 Guéguen P, Galinat H, Blouch MT, et al. Biological determinants of bleeding in patients with heterozygous factor XI deficiency. Br J Haematol 2012;156(02):245-251

41 Rugeri L, Quélin F, Chatard B, De Mazancourt P, Negrier C, Dargaud Y. Thrombin generation in patients with factor XI deficiency and clinical bleeding risk. Haemophilia 2010;16(05):771-777

42 Pike GN, Cumming AM, Hay CR, Bolton-Maggs PH, Burthem J. Sample conditions determine the ability of thrombin generation parameters to identify bleeding phenotype in FXI deficiency. Blood 2015;126(03):397-405

43 Livnat T, Shenkman B, Martinowitz U, et al. The impact of thrombin generation and rotation thromboelastometry on assessment of severity of factor XI deficiency. Thromb Res 2015;136 (02):465-473

44 Bertaggia Calderara D, Zermatten MG, Aliotta A, et al. . Tissue Factor-Independent Coagulation Correlates with Clinical Pheno- type in Factor XI Deficiency and Replacement Therapy. Thromb Haemost 2020. doi:10.1055/s-0040-1715899

$45 \mathrm{Al}$ Dieri R, Peyvandi F, Santagostino E, et al. The thrombogram in rare inherited coagulation disorders: its relation to clinical bleeding. Thromb Haemost 2002;88(04):576-582

46 James P, Salomon O, Mikovic D, Peyvandi F. Rare bleeding disorders - bleeding assessment tools, laboratory aspects and phenotype and therapy of FXI deficiency. Haemophilia 2014;20 (Suppl 4):71-75

47 Hansson KM, Nielsen S, Elg M, Deinum J. The effect of corn trypsin inhibitor and inhibiting antibodies for FXIa and FXIIa on coagulation of plasma and whole blood. J Thromb Haemost 2014;12(10): 1678-1686

48 Keularts IM, Zivelin A, Seligsohn U, Hemker HC, Béguin S. The role of factor XI in thrombin generation induced by low concentrations of tissue factor. Thromb Haemost 2001;85(06):1060-1065

49 Pike GN, Cumming AM, Thachil J, Hay CRM, Bolton-Maggs PHB, Burthem J. Evaluation of the use of rotational thromboelastometry in the assessment of FXI deficiency. Haemophilia 2017;23 (03):449-457

50 Gidley GN, Holle LA, Burthem J, Bolton-Maggs PHB, Lin FC, Wolberg AS. Abnormal plasma clot formation and fibrinolysis reveal bleeding tendency in patients with partial factor XI deficiency. Blood Adv 2018;2(10):1076-1088

51 Colucci M, Incampo F, Cannavò A, et al. Reduced fibrinolytic resistance in patients with factor XI deficiency. Evidence of a thrombin-independent impairment of the thrombin-activatable fibrinolysis inhibitor pathway. J Thromb Haemost 2016;14(08): 1603-1614

52 Boffa MB. Is resistance futile? The role of activated thrombinactivatable fibrinolysis inhibitor resistance in bleeding in factor XI deficiency. J Thromb Haemost 2016;14(08):1600-1602

53 Soshitova NP, Karamzin SS, Balandina AN, et al. Predicting prothrombotic tendencies in sepsis using spatial clot growth dynamics. Blood Coagul Fibrinolysis 2012;23(06):498-507

54 Parunov LA, Fadeeva OA, Balandina AN, et al. Improvement of spatial fibrin formation by the anti-TFPI aptamer BAX499: changing clot size by targeting extrinsic pathway initiation. J Thromb Haemost 2011;9(09):1825-1834

55 Ovanesov MV, Krasotkina JV, Ul'yanova LI, et al. Hemophilia A and $B$ are associated with abnormal spatial dynamics of clot growth. Biochim Biophys Acta 2002;1572(01):45-57

56 Dashkevich NM, Ovanesov MV, Balandina AN, et al. Thrombin activity propagates in space during blood coagulation as an excitation wave. Biophys J 2012;103(10):2233-2240

57 Pike GN, Cumming AM, Thachil J, Hay CR, Burthem J, BoltonMaggs PH. Evaluation of the use of global haemostasis assays to monitor treatment in factor XI deficiency. Haemophilia 2017;23 (02):273-283

58 Pike GN, Cumming AM, Hay CR, et al. In vitro comparison of the effect of two factor XI (FXI) concentrates on thrombin generation in major FXI deficiency. Haemophilia 2016;22(03):403-410

59 Bouma BN, von dem Borne PA, Meijers JC. Factor XI and protection of the fibrin clot against lysis-a role for the intrinsic pathway of coagulation in fibrinolysis. Thromb Haemost 1998;80(01):24-27

60 Vandenbroucke JP, Rosing J, Bloemenkamp KW, et al. Oral contraceptives and the risk of venous thrombosis. N Engl J Med 2001;344 (20):1527-1535

61 Weidmann H, Heikaus L, Long AT, Naudin C, Schlüter H, Renné T. The plasma contact system, a protease cascade at the nexus of inflammation, coagulation and immunity. Biochim Biophys Acta Mol Cell Res 2017;1864(11, Pt B):2118-2127 